\title{
The impact of omeprazole on mycophenolate pharmacokinetics in kidney transplant recipients
}

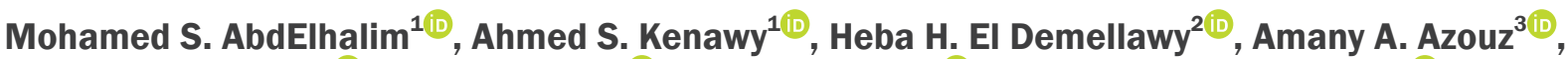 \\ Sarah S. Alghanem ${ }^{4 \mathbb{D}}$, Torki Al-Otaibi ${ }^{1 \mathbb{D}}$, Osama Gheith ${ }^{1,5}{ }^{\mathbb{D}}$, Mohamed Abd ElMonem $^{1 \mathbb{D}}$, \\ Mohammed K. Afifi ${ }^{1}$, Raghda R. S. Hussein ${ }^{6}$ iD \\ 'Hamed Al-Essa Organ Transplant Center, Ibn Sina Hospital, Kuwait City, Kuwait \\ ${ }^{2}$ Department of Nephrology, Faculty of Medicine, Beni-Suef University, Beni Suef, Egypt \\ ${ }^{3}$ Department of Pharmacology and Toxicology, Faculty of Pharmacy, Beni-Suef University, Beni-Suef, Egypt \\ ${ }^{4}$ Department of Pharmacy Practice, Faculty of Pharmacy, Kuwait University, Kuwait City, Kuwait \\ ${ }^{5}$ Urology and Nephrology Center, Mansoura University, Mansoura, Egypt \\ ${ }^{6}$ Department of Clinical Pharmacy, Faculty of Pharmacy, Beni-Suef University, Beni-Suef, Egypt
}

Background: The absorption rates of mycophenolate mofetil (MMF) and enteric-coated mycophenolate sodium (ECMPS) may be influenced by the concomitant use of omeprazole.

Methods: One hundred kidney transplant patients were recruited during their outpatient visits, including 50 on MMF and 50 on EC-MPS. At the clinic, a predose mycophenolic acid (MPA) sample $\left(\mathrm{C}_{0}\right)$ was collected; subsequently, the participants received the proton-pump inhibitor omeprazole along with either MMF or EC-MPS. Two more blood samples were collected at 1.5 and 3.5 hours and used to estimate an area under the curve (AUC) from zero to 12 hours [AUC (0-12)].

Results: The mean number of months after transplant was 92 months. The median AUC (0-12) and $C_{0}$ results were $62.2 \mathrm{mg} / \mathrm{h} / \mathrm{L}$ and $2.0 \mathrm{mg} / \mathrm{L}$ for the MMF group and $71.9 \mathrm{mg} \cdot \mathrm{h} / \mathrm{L}$ and $1.8 \mathrm{mg} / \mathrm{L}$ for the EC-MPS group $(P=0.160$ and 0.225 , respectively). Interestingly, $54 \%$ of the MMF group and $62 \%$ of the EC-MPS group showed AUCs above the target values. The correlation between MPA $\mathrm{C}_{0}$ and the predicted AUC was poor in both groups.

Conclusion: Omeprazole can be safely co-administered with either MMF or EC-MPS, as it did not compromise the MPA exposure. Unexpectedly, however, a high percentage of patients presented MPA AUCs exceeding the target value, highlighting the importance of periodically assessing MPA level.

Keywords: Area under curve, Enteric-coated mycophenolate sodium, Kidney transplantation, Mycophenolate mofetil, Mycophenolic acid, Omeprazole

Received April 21, 2020; Revised August 15, 2020; Accepted August 19, 2020

Editor: Byung Ha Chung, The Catholic University of Korea, Seoul, Republic of Korea

Correspondence: Raghda R. S. Hussein

Department of Clinical Pharmacy, Faculty of Pharmacy, Beni-Suef University, 55 Elshaheed Hegazi Street, Beni-Suef 62511, Egypt.

E-mail: raghda.hussien@pharm.bsu.edu.eg

Copyright (C) 2020 by The Korean Society of Nephrology

(a) This is an open-access article distributed under the terms of the Creative Commons Attribution Non-Commercial License (http://creativecommons.org/ licenses/by-nc-nd/4.0/), which permits unrestricted non-commercial use, distribution, and reproduction in any medium, provided the original work is properly cited. 


\section{Introduction}

Since 1995, mycophenolic acid (MPA) has been a standard agent in the prevention of organ-transplant rejection [1]. Currently, two mycophenolate formulations are available: mycophenolate mofetil (MMF) and entericcoated mycophenolate sodium (EC-MPS). Following administration, both are rapidly hydrolyzed to MPA as the active entity $[2,3]$. However, though both formulations release equivalent MPA amounts, they have different pharmacokinetic characteristics. MMF is the 2-morpholino ethyl ester of MPA, which displays high solubility in acidic media; in this context, the $\mathrm{C}_{\max }(24.1 \mathrm{mg} / \mathrm{L})$ is reached within 54 minutes $\left(\mathrm{T}_{\max }\right)$. Its $\mathrm{C}_{\max }$ is decreased by $40 \%$ if taken with food and it presents a bioavailability (F) of $94 \%$ [4]. Meanwhile, the enteric-coated sodium salt of MPA is a delayed-release tablet that is formulated to facilitate absorption in the intestine at a $\mathrm{pH}$ of greater than 5. The maximum concentration of EC-MPS is $24.1 \mathrm{mg} / \mathrm{L}$, which is reached two hours after intake. Its $\mathrm{C}_{\max }$ is not affected by food and it presents an F of 72\% [5-7].

The pharmacokinetics (PK) of MPA are characterized by considerable inter- and intrapatient variability and a significant correlation has been reported to exist between drug exposure and the risk of rejection and side effects $[2,8]$. MPA area under the curve (AUC) values of between 30 and $60 \mathrm{mg} \cdot \mathrm{hr} / \mathrm{L}$ have been proposed to constitute the target therapeutic window [9]. Meanwhile, MPA AUC values less than $30 \mathrm{mg} \cdot \mathrm{hr} / \mathrm{L}$ have been associated with significantly more acute rejections relative to values of 30 $\mathrm{mg} \cdot \mathrm{hr} / \mathrm{L}$ or greater $[9,10]$.

The success of the transplantation process depends upon the correct and continuous use of immunosuppressants and other medications. These medications can cause some unwanted drug-drug interactions that may lead to an increase or decrease in the blood concentration of the immunosuppressant. On the other hand, it is crucial to use these medications to reduce any unfavorable side effects associated with the treatment process to prevent early discontinuation of immunosuppressants, which causes more than $30 \%$ of graft losses in renal transplant patients $[11,12]$.

Omeprazole is a proton-pump inhibitors (PPIs) that is widely used to treat hyperacidity and other gastrointestinal complications associated with the use of immunosuppressants, especially corticosteroids. PPIs interact with many medications because of their effect on the stomach $\mathrm{pH}$, which may alter the absorption of an immunosuppressant agent, affecting the transplanted kidney [13-15]. As described in previous reports, the significance and effect of the interaction may change depending on the PPI in use. Miura et al [16] observed that both the MPA AUC and the MPA plasma concentration significantly decreased with the use of lansoprazole in renaltransplant patients. On the other hand, the use of pantoprazole did not alter the pharmacokinetic parameters of MPA in the study by Rupprecht et al [17]. Meanwhile, in a direct comparison between MMF and the enteric-coated formulation in Chinese patients, omeprazole was found to significantly decrease the $\mathrm{C}_{\max }, \mathrm{T}_{\max }$ and AUC MPA of MMF-treated patients and had no significant effect on EC-MPS pharmacokinetic parameters [18]. This interaction could potentially place patients at risk of acute rejection if therapeutic MPA exposures are not achieved.

The main immunosuppressant protocol at the local center includes prednisolone, tacrolimus, omeprazole $20 \mathrm{mg}$ twice daily and either MMF or EC-MPS. Therefore, the current study aimed to compare the impact of concomitant use of omeprazole on the MPA exposure by assessing the AUC from zero to 12 hours [AUC (0-12)] and $\mathrm{C}_{0}$ in patients treated with MMF (CellCept; Genentech, San Francisco, CA, USA) or EC-MPS (Myfortic; Novartis, Basel, Switzerland). Furthermore, it aimed to assess the attainment of MPA AUC (0-12) target levels for both formulations.

\section{Methods}

\section{Ethics approval}

The Ministry of Health Ethical Committee of Kuwait approved the study protocol (registration no. 2017/619).

\section{Study design}

A cross-sectional nonrandomized study was carried out in the outpatient department at Hamed Al-Essa Organ Transplant Center in Kuwait. The study protocol was approved by the ethical committee of the Ministry of Health, Kuwait, following the guidelines of the Declaration of Helsinki before the study was begun. Participants were considered eligible for the study if they were aged 21 
AbdElhalim, et al. Mycophenolate and omeprazole pharmacokinetics

years or older, presenting six months or more after transplant with stable renal function and with the ability to read and sign the informed consent form. Patients with one or more of the following conditions were excluded from the study: severe active infection requiring reduction or discontinuation of mycophenolate, use of histamine-2 receptor blockers or other PPIs, advanced renal dysfunction (i.e., glomerular filtration rate $<40 \mathrm{~mL} / \mathrm{min}$ as detected by the Modification of Diet in Renal Disease formula [19-23]) and/or using cyclosporine instead of tacrolimus as an immunosuppressant.

\section{Study protocol and sample collection}

The routine procedure at the study center is to collect blood samples one day before a scheduled outpatient visit. Weekly, the patient list was screened to select those patients whose characteristics were compatible with the study design. During the day of each patient's follow-up visit, study investigators explained the study objectives to the participants and obtained their approval for inclusion by having them sign the informed consent form. Participants were asked to come the next day having fasted overnight and without taking their morning medication doses.

The next day, a predose MPA sample was collected and, then, participants received their omeprazole dose (Minisec, 20 mg; Kuwait Saudi Pharmaceutical, Subhan Industrial Area, Kuwait) along with either MMF (CellCept, 500 $\mathrm{mg}$ or $250 \mathrm{mg}$ ) or EC-MPS (Myfortic, $360 \mathrm{mg}$ or $180 \mathrm{mg}$ ). Two more blood samples were collected at $1.5(\mathrm{Cl} .5)$ and 3.5 (C3.5) hours postdosing. The collected samples were analyzed by the Hamed Al-Essa laboratory, using a fully automated immunoassay cobas c 501 analyzer (Roche Diagnostics, Basel, Switzerland) to measure the serum MPA concentration in the blood. The samples were analyzed at once, with the serum stored in the fridge at a temperature between $2^{\circ} \mathrm{C}$ and $8^{\circ} \mathrm{C}$ for one week in case of the need to retest if necessary.

AUC calculation and statistical analysis: As the patients stayed at the clinic for a maximum of four hours during their outpatient visit, the MPA AUC (0-12) was calculated using a limited sampling strategy based on multiple linear regressions as per the research by Musuamba et al [24]. MPA levels at C1.5 and C3.5 were used in the model equation AUC $(0-12)=16.5+4.9 \times \mathrm{C} 1.5+6.7 \times \mathrm{C} 3.5$ to predict the MPA AUC.
Table 1. Baseline characteristics of the MMF and EC-MPS groups

\begin{tabular}{lccc}
\hline \multicolumn{1}{c}{ Parameter } & MMF & EC-MPS & $P$ value \\
\hline No. of patients & 50 & 50 & - \\
Male/female & $38 / 12$ & $28 / 22$ & $0.035^{\mathrm{b}}$ \\
Age (yr) & $48 \pm 15$ & $44.5 \pm 13$ & $0.049^{\mathrm{b}}$ \\
Months after transplant & $115 \pm 71.5$ & $84 \pm 59$ & $0.028^{\mathrm{b}}$ \\
Mean tacrolimus trough level & $5.89 \pm 1.42$ & $6.15 \pm 0.94$ & $0.550^{\mathrm{a}}$ \\
(ng/mL) & & & \\
Daily dose (MMF/EC-MPS) & & & $0.042^{\mathrm{b}}$ \\
$\quad$ 1,000 mg/720 mg & $7(14 \%)$ & $16(32 \%)$ & \\
1,500 mg/1,080 mg & $23(46 \%)$ & $24(48 \%)$ & \\
$2,000 \mathrm{mg} / 1,440 \mathrm{mg}$ & $20(40 \%)$ & $10(20 \%)$ & \\
\hline
\end{tabular}

Data are presented as number only, mean \pm standard deviation, or percentages only.

Categorical data are presented as percentages and were compared using the chi-squared test, while the Mann-Whitney $U$ test was used for continuous variables; significance was found at $P \leq 0.05$.

EC-MPS, enteric-coated mycophenolate sodium; MMF, mycophenolate mofetil.

${ }^{a}$ Nonsignificant difference between the two groups. ${ }^{b}$ Significant difference between the two groups.

Statistical analysis was conducted using the IBM SPSS Statistics ver. 24.0 software program (IBM Corp., Armonk, NY, USA). Data were not normally distributed and, hence, values of continuous variables were expressed as median and ranges. The Mann-Whitney $U$ test was used for continuous variables to compare between the studied groups. Categorical data were presented as percentages and compared using the chi-squared test. Statistical significance was set at $P \leq 0.05$.

\section{Results}

One hundred fifty-five patients were eligible for inclusion in this study and were invited to participate; ultimately, 135 patients accepted the invitation and $100 \mathrm{com}$ pleted the study. Baseline characteristics for the study participants are presented in Table 1. An equal number of patients received MMF and EC-MPS, with a total of 50 participants included in each group, respectively. More than $50 \%$ of the patients were male in both groups and the majority received $1,500 \mathrm{mg}$ or $1,080 \mathrm{mg}$ per day of MMF or EC-MPS. There were no statistically significant differences in laboratory results between both groups.

MPA exposure levels with the measured concentrations for the MMF and EC-MPS groups are shown in Table 2. There were no statistically significant differences in the 
Table 2. MPA exposures with measured concentrations for the two groups

\begin{tabular}{lccr}
\hline \multicolumn{1}{c}{ Parameter } & MMF & EC-MPS & \multicolumn{1}{c}{$P$ value } \\
\hline C $_{0}(\mathrm{mg} / \mathrm{L})$ & $2.00(1.93)$ & $1.80(1.30)$ & $0.225^{\mathrm{a}}$ \\
C1.5 $(\mathrm{mg} / \mathrm{L})$ & $4.48(3.42)$ & $3.41(3.61)$ & $0.049^{\mathrm{b}}$ \\
C3.5 $(\mathrm{mg} / \mathrm{L})$ & $3.29(2.60)$ & $4.90(3.95)$ & $<0.001^{\mathrm{b}}$ \\
AUC $(0-12)$ & $62.21(20.29)$ & $71.88(43.80)$ & $0.160^{\mathrm{a}}$ \\
\hline
\end{tabular}

Data are presented as median (interquartile range).

AUC, area under the curve; $C_{0}$, MPA concentration at zero time (predose); $\mathrm{C} 1.5$, MPA concentration at 1.5 hours after $\mathrm{C}_{0} ; \mathrm{C} 3.5$, MPA concentration at 3.5 hours after $\mathrm{C}_{0}$; EC-MPS, enteric-coated mycophenolate sodium; MMF, mycophenolate mofetil; MPA, mycophenolic acid.

The Mann-Whitney $U$ test was used; significance was found at $P \leq 0.05$.

${ }^{a}$ Nonsignificant difference between the two groups. ${ }^{b}$ Significant difference between the two groups.

Table 3. The percentages of patients whose AUC (0-12) estimates were below, within and above the target ranges, respectively, when using MMF and EC-MPS

\begin{tabular}{cccc}
\hline AUC $(0-12)(\mathrm{mg} \cdot \mathrm{h} / \mathrm{L})$ & MMF & EC-MPS & $P$ value \\
\hline$<30$ & 0 & $2 \%$ & \\
$30-60$ & $46 \%$ & $36 \%$ & $0.252^{\mathrm{a}}$ \\
$>60$ & $54 \%$ & $62 \%$ & \\
\hline
\end{tabular}

The target range of MPA is from 30 to $60 \mathrm{mg} / \mathrm{h}$.

AUC, area under the curve; EC-MPS, enteric-coated mycophenolate sodium; MMF, mycophenolate mofetil; MPA, mycophenolic acid.

${ }^{a}$ Nonsignificant difference between the two groups, using the chi-square test.

MPA AUC (0-12) and $\mathrm{C}_{0}$ values between the MMF and EC-MPS groups $(P=0.160$ and 0.225 , respectively). However, the MMF group showed significantly higher C1.5 values than the EC-MPS group $(P=0.049)$, while the ECMPS group presented significantly higher C3.5 values relative to the MMF group $(P<0.001)$.

The percentages of patients whose AUC (0-12) estimates were below, within and above the target ranges associated with MMF and EC-MPS, respectively, are reported in Table 3. A target MPA AUC (0-12) range of 30 to $60 \mathrm{mg} \cdot \mathrm{hr} / \mathrm{L}$ was achieved in $46 \%$ and $36 \%$ of the MMF and EC-MPS groups, respectively. A large proportion of patients presented MPA AUC (0-12) values of more than $60 \mathrm{mg} \cdot \mathrm{hr} / \mathrm{L}$ (54\% and $62 \%$ in MMF and EC-MPS groups, respectively; $P=0.252$ ).

The numbers and percentages of patients who had an MPA AUC (0-12) value of greater than $60 \mathrm{mg} \cdot \mathrm{hr} / \mathrm{L}$ are presented in Fig. 1 . More than $80 \%$ of patients who received 1,000 mg of MMF had AUC (0-12) value of greater than $60 \mathrm{mg} \cdot \mathrm{hr} / \mathrm{L}$ as compared with $56.25 \%$ with equiva-
Total number pf the patients ${ }^{\mathrm{a}}$

Number of the patients who had AUC > $60 \mathrm{mg} \cdot \mathrm{hr}^{\mathrm{b}} \mathrm{L}^{\mathrm{b}}$

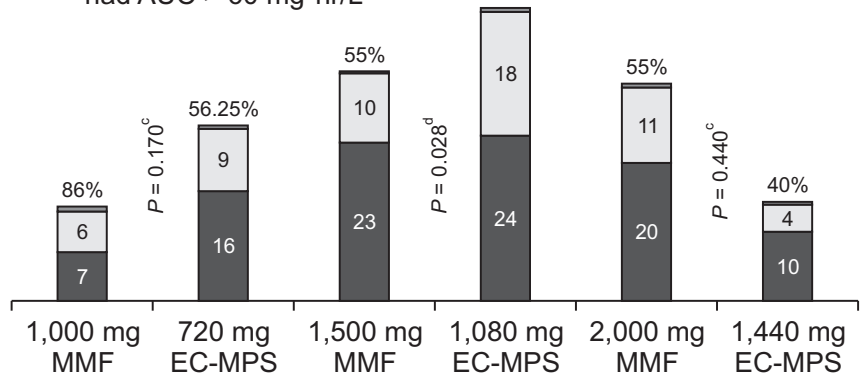

Figure 1. The numbers and the percentage of recipients with MPA-AUC $>60 \mathrm{mg} \cdot \mathrm{hr} / \mathrm{L}$ to the total number of the recipients who receive equivalent doses.

AUC, area under the curve; EC-MPS, enteric-coated mycophenolate sodium; MMF, mycophenolate mofetil; MPA, mycophenolic acid.

${ }^{a}$ The total number of patients who receive a specific dose of either MMF \& EC-MPS. ${ }^{\text {T}}$ The number of patients who had AUC values of more than $60 \mathrm{mg} \cdot \mathrm{h} / \mathrm{L}$ (above the targeted AUC) of the same dose. MMF doses are 1,000 mg, 1,500 mg and 2,000 mg. EC-MPS doses are $720 \mathrm{mg}, 1,080 \mathrm{mg}$ and 1,440 mg. 'Nonsignificant difference between the two groups. 'Significant difference between the two groups.

Table 4. Degree of the correlation between MPA concentration and AUC level

\begin{tabular}{cccccc}
\hline \multirow{2}{*}{ Parameter } & \multicolumn{2}{c}{ MMF AUC $(0-12)$} & & \multicolumn{2}{c}{ EC-MPS AUC $(0-12)$} \\
\cline { 2 - 3 } \cline { 5 - 6 } & \multicolumn{1}{c}{$r$} & $P$ value & & $r$ & $P$ value \\
\hline $\mathrm{C}_{0}$ MPA & -0.075 & $0.621^{\mathrm{a}}$ & & 0.498 & $0.001^{\mathrm{b}}$ \\
\hline
\end{tabular}

AUC, area under the curve; CO, MPA concentration at zero time (predose); ECMPS, enteric-coated mycophenolate sodium; MMF, mycophenolate mofetil; MPA, mycophenolic acid; r, correlation.

Pearson's correlation test was used; correlation was poor in both groups.

${ }^{a}$ No significant correlation between AUC and $\mathrm{C}_{0}$ MPA. ${ }^{\mathrm{b}}$ Significant correlation between $A U C$ and $C_{0}$ MPA.

lent EC-MPS dosing $(P=0.170)$. Meanwhile, 18 patients (75\%) who received $1,080 \mathrm{mg}$ of EC-MPS had AUC (012) values above the target range in comparison with 10 patients $(43.48 \%)$ on $1,500 \mathrm{mg}$ of $\mathrm{MMF}(P=0.028)$. In the 2,000-mg MMF and 1,440-mg EC-MPS groups, the percentages were $55 \%$ and $40 \%$, respectively $(P=0.440)$.

The correlation between MPA $\mathrm{C}_{0}$ and the predicted AUC is shown in Table 4. A poor correlation was found to exist between the variable in the two groups, with no statistically significant difference.

\section{Discussion}

The current study was conducted to evaluate the AUC and $\mathrm{C}_{0}$ of both MMF and EC-MPS with the concomitant 
use of omeprazole. The results suggested the absence of any significant difference in both AUC $(0-12)$ and $C_{0}$ between the groups $(P>0.05)$, which indicates that stable renal transplant recipients who use omeprazole could be safely shifted between MMF and EC-MPS if needed. At the same time, the concentration of MPA at C1.5 was significantly higher in the MMF group but was higher at C3.5 in the EC-MPS group $(P=0.049$ and $P<0.001$, respectively).

The AUC (0-12) is a decisive parameter by which to evaluate patient exposure to MPA as it is directly related to unwanted effects and medication outcomes [25]. To accurately calculate it, at least 10 blood samples much be collected over 12 hours; thus, this method is costly and difficult to adopt, especially in outpatient departments. As such, limited sampling strategies and pharmacokinetic models have emerged that are simpler to use to estimate the MPA AUC in such settings [26-28].

Musuamba et al [24] previously developed a multiple linear regression model for monitoring MPA concomitantly administered with tacrolimus within four hours following dose administration. In their study, these authors compared the outcomes of many prediction models to the measured AUCs in transplant patients receiving either MMF or EC-MPS along with tacrolimus [24] and concluded that the equation AUC $(0-12)=16.5+4.9 \times \mathrm{C} 1.5+6.7 \times$ C3.5, with a regression coefficient $\left(\mathrm{r}^{2}\right)$ of 0.82 , is the most accurate and precise model by which to predict MPA total exposure. In the Hamed Al-Essa outpatient clinic, it was deemed appropriate to use this equation to predict the MPA AUC during the scheduled outpatient appointments.

As a PPI, omeprazole increases the gastric $\mathrm{pH}$, which might affect the absorption process of co-administered oral medications, especially those that are formulated to be absorbed in low $\mathrm{pH}$ conditions like MMF. On the other hand, the effect of PPI was expected to be diminished in correlation with intestine-absorbed drugs like EC-MPS. Previous results on the effect of omeprazole on MPA $\mathrm{PK}$ are conflicting. For instance, in the study by Xu et al [18], omeprazole was reported to significantly decrease the MPA AUC by at least $35 \%$ during co-administration with MMF as compared with EC-MPS. Meanwhile, in the study by Fernandez-Rivera et al [29], both MMF and ECMPS patients showed greater exposure to MPA with the use of omeprazole as compared with in nonomeprazole patients. In the current study, the EC-MPS group showed nonsignificantly higher MPA AUC values when compared with those of the MMF group, which were comparable to those reported by David-Neto et al [30].

David-Neto et al [30] also mentioned in their analysis that the reduction effect of omeprazole on MPA PK can be observed only in the first week during the first year of transplantation, thereafter becoming clinically irrelevant. As such, the time after transplantation could be used as an explanation for the discrepancies observed between the results of previous studies. For example, omeprazole significantly affected MPA pharmacokinetic parameters in the study by Xu et al [18], who investigated MPA samples from newly transplanted patients, while this study and that by Fernandez-Rivera et al [29] assessed the effect after six months of transplantation; in particular, the mean time after transplant in this study was more than 80 months in both groups.

Another crucial factor that might explain the variations in the results is related to the omeprazole brand in use. Shimatani et al [31] concluded that the acid-suppression effect of some brands of generic omeprazole was different from that of the original product, Moreover, Okorie et al [32] concluded that the brand of an omeprazole medication had a significant influence on its release rate and may therefore impact clinical outcomes. As such, the patients who are co-medicated with a generic substitution for drugs such as chemotherapeutic drugs and drugs with narrow therapeutic indices should be closely monitored. Consequently, the use of different omeprazole generics may affect the extent of MMF dissolution and, thus, its pharmacokinetic parameters. Previous studies did not mention the brand of administered omeprazole generic; while, in this study, omeprazole (Minisec, $20 \mathrm{mg}$ ) was used throughout the study period.

As expected, the C1.5 value was higher in the MMF group and the C3.5 value was higher in the EC-MPS group, respectively. This can be explained by differences in the site of absorption of each drug, as MMF is absorbed from the stomach and reaches its maximum concentration in the blood after about one hour, while EC-MPS is absorbed from the intestine and reaches its maximum concentration in the blood after 1.5 to 2.75 hours [4-7].

Recently, many studies have highlighted the strong correlation between the level of MPA and its pharmaco- 
logical efficacy and toxicity, especially given the strong interindividual variability noted in the pharmacokinetic parameters between patients given the same dose. Therefore, MPA monitoring is required to optimize its exposure and dosing. A consensus guideline recommended monitoring MPA with the AUC (0-12) approach and considering an ideal target range of 30 to $60 \mathrm{mg} \cdot \mathrm{hr} / \mathrm{L}[10,33,34]$. In the present study, more than $50 \%$ of patients in both groups had an AUC (0-12) MPA level of $60 \mathrm{mg} \cdot \mathrm{h} / \mathrm{L}$ or more, exceeding the target range. This outcome necessitates the revision of the transplant center protocol that uses MMF and EC-MPS in fixed daily doses and supports the necessity of adopting MPA therapeutic drug monitoring and treatment personalization in the near future.

The time-dependent characteristic of AUC MPA is another reason suggesting the need to re-evaluate the MPA exposure after six months of transplantation. The center protocol should be reviewed because the mean AUC (012) of MPA in the late posttransplantation (3-6 months) is almost $40 \%$ higher for the same dose than that in the early posttransplantation period $[35,36]$. It is also important to note that omeprazole may have a role in the presence of such high MPA AUC levels as per the research by Fernandez-Rivera et al [29].

Although the predose concentration $\mathrm{C}_{0}$ could be used to evaluate drug exposure, its application is weak due to its poor correlation with the MPA AUC, which is considered a better predictor of MPA clinical outcomes. As seen in Table 4, the correlation between $\mathrm{MPA} \mathrm{C}_{0}$ and predicted AUC was investigated and a poor correlation in both groups was noted $(r=-0.075$ for MMF and $r=0.498$ for EC-MPS). With the use of both MPA and tacrolimus, a $\mathrm{C}_{0}$ of at least $1.9 \mathrm{mg} / \mathrm{L}$ of MPA is required to attain an MPA AUC value within the target level. At the same time, a $\mathrm{C}_{0}$ of $2.75 \mathrm{mg} / \mathrm{L}$ or more is associated with more frequent side effects like diarrhea and hematological toxicity $[34,37]$. Twenty-three patients in both groups had a $\mathrm{C}_{0}$ of at least $2.75 \mathrm{mg} / \mathrm{L}(17 \mathrm{mg} / \mathrm{L}$ in the MMF group and $6 \mathrm{mg} /$ $\mathrm{L}$ in the EC-MPS group), warranting further attention in the future.

This study had some limitations, the first of which was the absence of a control group (i.e., inclusion of patients not on omeprazole). The second limitation was the performance of an analysis of pharmacokinetic parameters only, without relating to clinical outcomes including graft rejection and side effects. Additionally, this study used a limited sample strategy for AUC estimation that is considered to be less accurate than the 12-hour samplecollection method. Another limitation is the variations in sex, age and posttransplant duration between the study groups. Previous studies have clarified that these factors do not change the pharmacokinetic parameters significantly. Pescovitz et al [38] concluded that there was no significant difference in the PK of MMF between males and females. At the same time, age did not significantly affect the PK or pharmacodynamics (PD) of MPA in the research by Tang et al [39]. Meanwhile, Mohammadpour et al [40] observed that the MPA AUC and clearance were not affected by the time after transplantation.

According to our results, the concomitant use of omeprazole with MPA formulations (MMF or EC-MPS) did not result in different exposure levels or predose concentrations in late transplant patients. Omeprazole can be coadministered with MPA (MMF or EC-MPS) without compromising MPA exposure. However, the analysis of MPA exposure indicated unexpectedly high exposure levels with the use of both formulations. We recommend the use of individualized MPA dosing instead of fixed daily dosing and the pursuit of further investigation to elucidate the reasons behind these results.

\section{Conflicts of interest}

All authors have no conflicts of interest to declare.

\section{Acknowledgments}

The authors are truly grateful to Dr. Prasad Nair, Dr. Tarek Said, Dr. Medhat Abdel Halim, Dr. Khaled Abdel Tawab, Dr. Ayman Naguib, Ms. Najat Al-Ali, and the nursing staff at the Hamed Al-Essa Center for Organ Transplantation for their support and help during this research.

\section{Authors' contributions}

Mohamed S. AbdElhalim, Ahmed S. Kenawy, and Mohammed K. Afifi participated in the data collection and wrote the manuscript. Mohamed S. AbdElhalim, Amany A. Azouz, Raghda R. S. Hussein, Ahmed S. Kenawy, Heba H. El Demellawy, and Osama Gheith participated in the study design and performed the statistical analysis. 
Sarah S. Alghanem, Mohamed S. AbdElhalim, Ahmed S. Kenawy, and Raghda R. S. Hussein participated in the conception, analysis, and interpretation of data. Heba H. El Demellawy, Torki Al-Otaibi, Amany A. Azouz, and Mohamed Abd ElMonem provided intellectual content of critical importance to the work and technical support. Sarah S. Alghanem, Mohamed Abd ElMonem, and Mohammed K. Afifi participated in the study design and coordination and helped to draft the manuscript. All authors read and approved the final manuscript.

\section{References}

[1] Bentley R. Mycophenolic acid: a one hundred year odyssey from antibiotic to immunosuppressant. Chem Rev 2000; 100:3801-3826.

[2] Staatz CE, Tett SE. Clinical pharmacokinetics and pharmacodynamics of mycophenolate in solid organ transplant recipients. Clin Pharmacokinet 2007;46:13-58.

[3] Cholewinski G, Malachowska-Ugarte M, Dzierzbicka K. The chemistry of mycophenolic acid--synthesis and modifications towards desired biological activity. Curr Med Chem 2010;17:1926-1941.

[4] Bullingham RE, Nicholls AJ, Kamm BR. Clinical pharmacokinetics of mycophenolate mofetil. Clin Pharmacokinet 1998;34:429-455.

[5] Budde K, Bauer S, Hambach P, et al. Pharmacokinetic and pharmacodynamic comparison of enteric-coated mycophenolate sodium and mycophenolate mofetil in maintenance renal transplant patients. Am J Transplant 2007;7: 888-898.

[6] Johnson AG, Rigby RJ, Taylor PJ, et al. The kinetics of mycophenolic acid and its glucuronide metabolite in adult kidney transplant recipients. Clin Pharmacol Ther 1999;66: 492-500.

[7] Arns W, Breuer S, Choudhury S, et al. Enteric-coated mycophenolate sodium delivers bioequivalent MPA exposure compared with mycophenolate mofetil. Clin Transplant 2005;19:199-206.

[8] van Gelder T, Hilbrands LB, Vanrenterghem Y, et al. A randomized double-blind, multicenter plasma concentration controlled study of the safety and efficacy of oral mycophenolate mofetil for the prevention of acute rejection after kidney transplantation. Transplantation 1999;68:261-266.

[9] Kuypers DR, Le Meur Y, Cantarovich M, et al.; Transplantation Society (TTS) Consensus Group on TDM of MPA.
Consensus report on therapeutic drug monitoring of mycophenolic acid in solid organ transplantation. Clin J Am Soc Nephrol 2010;5:341-358.

[10] Shaw LM, Figurski M, Milone MC, Trofe J, Bloom RD. Therapeutic drug monitoring of mycophenolic acid. Clin J Am Soc Nephrol 2007;2:1062-1072.

[11] Rathee P, Chaudhary H, Rathee S, Rathee D, Kumar V. Immunosuppressants: a review. Pharma Innov 2013;1:90-101.

[12] Davies NM, Grinyó J, Heading R, Maes B, Meier-Kriesche HU, Oellerich M. Gastrointestinal side effects of mycophenolic acid in renal transplant patients: a reappraisal. Nephrol Dial Transplant 2007;22:2440-2448.

[13] Shin JM, Kim N. Pharmacokinetics and pharmacodynamics of the proton pump inhibitors. J Neurogastroenterol Motil 2013;19:25-35.

[14] Li W, Zeng S, Yu LS, Zhou Q. Pharmacokinetic drug interaction profile of omeprazole with adverse consequences and clinical risk management. Ther Clin Risk Manag 2013;9: 259-271.

[15] Wedemeyer RS, Blume H. Pharmacokinetic drug interaction profiles of proton pump inhibitors: an update. Drug Saf 2014;37:201-211.

[16] Miura M, Satoh S, Inoue K, et al. Influence of lansoprazole and rabeprazole on mycophenolic acid pharmacokinetics one year after renal transplantation. Ther Drug Monit 2008; 30:46-51.

[17] Rupprecht K, Schmidt C, Raspé A, et al. Bioavailability of mycophenolate mofetil and enteric-coated mycophenolate sodium is differentially affected by pantoprazole in healthy volunteers. J Clin Pharmacol 2009;49:1196-1201.

[18] Xu L, Cai M, Shi BY, Li ZL, Li X, Jin HL. A prospective analysis of the effects of enteric-coated mycophenolate sodium and mycophenolate mofetil co-medicated with a proton pump inhibitor in kidney transplant recipients at a single institute in China. Transplant Proc 2014;46:1362-1365.

[19] Levey AS, Coresh J, Greene T, et al.; Chronic Kidney Disease Epidemiology Collaboration. Expressing the modification of diet in renal disease study equation for estimating glomerular filtration rate with standardized serum creatinine values. Clin Chem 2007;53:766-772.

[20] Stevens LA, Manzi J, Levey AS, et al. Impact of creatinine calibration on performance of GFR estimating equations in a pooled individual patient database. Am J Kidney Dis 2007;50:21-35.

[21] Imai E, Horio M, Nitta K, et al. Estimation of glomerular filtration rate by the MDRD study equation modified for 
Japanese patients with chronic kidney disease. Clin Exp Nephrol 2007;11:41-50.

[22] Masson I, Flamant M, Maillard N, et al. MDRD versus CKDEPI equation to estimate glomerular filtration rate in kidney transplant recipients. Transplantation 2013;95:12111217.

[23] Salvador CL, Hartmann A, Åsberg A, Bergan S, Rowe AD, Mørkrid L. Estimating glomerular filtration rate in kidney transplant recipients: comparing a novel equation with commonly used equations in this population. Transplant Direct 2017;3:e332.

[24] Musuamba FT, Mourad M, Haufroid V, et al. Statistical tools for dose individualization of mycophenolic acid and tacrolimus co-administered during the first month after renal transplantation. Br J Clin Pharmacol 2013;75:1277-1288.

[25] Pawinski T, Hale M, Korecka M, Fitzsimmons WE, Shaw LM. Limited sampling strategy for the estimation of mycophenolic acid area under the curve in adult renal transplant patients treated with concomitant tacrolimus. Clin Chem 2002;48:1497-1504.

[26] Jia Y, Peng B, Li L, et al. Estimation of mycophenolic acid area under the curve with limited-sampling strategy in Chinese renal transplant recipients receiving enteric-coated mycophenolate sodium. Ther Drug Monit 2017;39:29-36.

[27] Baraldo M, Cojutti PG, Isola M, et al. Validation of limited sampling strategy for estimation of mycophenolic acid exposure during the first year after heart transplantation. Transplant Proc 2009;41:4277-4284.

[28] Zhang J, Sun Z, Zhu Z, et al. Pharmacokinetics of mycophenolate mofetil and development of limited sampling strategy in early kidney transplant recipients. Front Pharmacol 2018;9:908.

[29] Fernandez-Rivera C, Salvador-Garrido P, Outeda-Macias $\mathrm{M}$, et al. Impact of omeprazole on mycophenolic acid pharmacokinetics in adult renal transplant patients.: Abstract\# C1785. Transplantation 2014;98:577.

[30] David-Neto E, Takaki KM, Agena F, et al. Diminished mycophenolic acid exposure caused by omeprazole may be clinically relevant in the first week posttransplantation. Ther Drug Monit 2012;34:331-336.
[31] Shimatani T, Inoue M, Kuroiwa T, Xu J, Mieno H, Tazuma S. Acid-suppressive effects of generic omeprazole: comparison of three brands of generic omeprazole with original omeprazole. Dig Liver Dis 2006;38:554-559.

[32] Okorie O, Azaka JE, Amadi CM. Pharmaceutical quality assurance of omeprazole capsule brands commonly used in health institutions in Southern Nigeria. Am J Pharm Pharmacol 2016;3:33-39.

[33] Yew SS, Caroll R, Tran H, Coates PT. Mycophenolic acid therapeutic drug monitoring in de novo renal transplant patients and the related outcomes. Transplantation 2018; 102:S129.

[34] Jeong H, Kaplan B. Therapeutic monitoring of mycophenolate mofetil. Clin J Am Soc Nephrol 2007;2:184-191.

[35] Luisa RPA, Alejandro MFM. Clinical pharmacokinetics of triple immunosuppression scheme in kidney transplant (tacrolimus, mycophenolate mofetil and corticosteroids). In: Ortiz J, Andre J, eds. Understanding the complexities of kidney transplantation. 6th ed. London: IntechOpen, 2011: 408-438.

[36] van Hest RM, van Gelder T, Bouw R, et al. Time-dependent clearance of mycophenolic acid in renal transplant recipients. Br J Clin Pharmacol 2007;63:741-752.

[37] Borrows R, Chusney G, Loucaidou M, et al. Mycophenolic acid 12-h trough level monitoring in renal transplantation: association with acute rejection and toxicity. Am J Transplant 2006;6:121-128.

[38] Pescovitz MD, Guasch A, Gaston R, et al. Equivalent pharmacokinetics of mycophenolate mofetil in African-American and Caucasian male and female stable renal allograft recipients. Am J Transplant 2003;3:1581-1586.

[39] Tang JT, de Winter BC, Hesselink DA, Sombogaard F, Wang LL, van Gelder T. The pharmacokinetics and pharmacodynamics of mycophenolate mofetil in younger and elderly renal transplant recipients. Br J Clin Pharmacol 2017;83: 812-822.

[40] Mohammadpour AH, Vahabzadeh M, Abedi H. Effect of time after transplantation on mycophenolic acid pharmacokinetic in kidney transplant patients in Iran: 326. Transplantation 2012;94:837. 